\title{
Image Registration using combination of PCA and GPOF Method for Multiframe Super-Resolution
}

\author{
Jagruti Rajput \\ PG Student \\ Parul Institute of Technology
}

\author{
Bhailal Limbasiya \\ Assistant Professor \\ Parul Institute of Technology
}

\begin{abstract}
Super-resolution technique is used for resolution enhancement. In Multiframe Super-resolution multiple low resolution images are combined to get high resolution image. Most important part of Multiframe Super-resolution is Image Registration that estimates translation,rotation and scaling parameters. In this paper they propose combination of PCA and GPOF registration method for constructing high resolution image. In GPOF registration method, which reach the sub pixel precision and allow large pixel motions and then apply PCA method which is used for compression of image. Image fusion is applied to get one output image. Then bicubic interpolation is used to get high resolved image. Experiment images show that the HR image by our proposed method has much higher quality than other methods.
\end{abstract}

\section{Keywords}

Spatial resolution; multiframe super-resolution; Gaussian Pyramid Optical Flow; Principal Component analysis; image fusion; Bicubic image interpolation

\section{INTRODUCTION}

Image Super-Resolution ${ }^{[1]}$ is most widely used research area and it can solve the limited resolution problem by image acquisition devices and sensor. But, high resolution sensor is very expensive. So, we can increase the resolution by two ways either by reducing pixel size or by increasing chip size. But it can generate noise and result in degradable image quality. So, there is another method to increase resolution of image. Super-resolution can be used in medical imaging, satellite imaging, remote imaging, enlarging consumer photograph for higher quality etc. Super-resolution techniques can be classified into two major parts: Frequency domain and spatial domain approach. Frequency domain approach can perform fourier transform of an image. These methods are simple and cheap. Spatial domain approach can perform directly on pixel. This method is popular but expensive.

Technical implementation of super-resolution can be done in two ways: single-frame and multi-frame. Single-frame superresolution methods generate high-resolution image from single degraded noisy image. Multi-frame super-resolution is to generate high-resolution image from multiple lowresolution images of same scene and increase spatial resolution by fusing information. Most important part of
Multiframe Super-resolution is Image Registration. There are many methods of image registration like Gradient, PCA and GPOF method. In Gradient method ${ }^{[3]}$, it can perform registration precisely only if the movement parameters are small. In GPOF method ${ }^{[4]}$, it can perform registration precisely by allowing large pixel motions. PCA method ${ }^{[5]}$ is used for compression of Image. Therefore our paper proposes combination of PCA and GPOF method for Multiframe Super-resolution. Here we apply GPOF method for large amount of movement parameters. Then apply PCA method for compression of image. Then Image fusion ${ }^{[6]}$ using simple averaging method is applied to get one output image. Finally Image interpolation using Bicubic interpolation ${ }^{[7]}$ is used to get high resolution image.

This paper is organized as follows. In Section 2, we describe multiframe super-resolution. Section 3 represents proposed work. Section 4 experiment results. Section 5 represents conclusion

\section{MULTIFRAME SUPER-RESOLUTION}

In Multi-frame SR imaging ${ }^{[2]}$, performing the observed LR image from SR image is modeled by:

$$
y=F H D_{x}+n
$$

Where, $\mathrm{x}$ is an original HR image, $\mathrm{F}$ is linear transformation, $\mathrm{H}$ is a image warping, $\mathrm{D}$ is the down-sampling operator and $\mathrm{n}$ is a noise. In this paper, multi-frame Super-Resolution is to generate the high-resolution (HR) image from multiple lowresolution images perspectives of a same scene and also increase spatial resolution by fusing information. There are three steps for multi-frame SR, 1) Image Registration, which is performed first for alignment of the LR images as accurately as possible, and also estimate movement parameters, 2) Image Fusion, which is the process of combing information of interest in two or more LR images into a single high-resolution image, 3) Interpolation, which is done to get high- resolved image.

Image registration plays accurate role in image reconstruction process. It is used for motion estimation like shift, rotation and scale estimation. There are three types of Registration parameters: Translation parameter, which calculates horizontal and vertical displacement between the reference image and changing image; Rotation parameter, which calculates the shift angle between two images; Scale 
parameter, which calculates size variance in same object. The image Registration methods are as below:

\subsection{GPOF Registration Method}

Gaussian Pyramid Optical Flow(GPOF) method which can perform sup-pixel precision and allows large pixel motions, which means if pixel points move very far, while keeping neighborhood of image relatively small.

In Gaussian Pyramid, we define no. of levels $\mathrm{K}$ like maximum no of levels 6 . Then build a pyramid g1 as base level image and downsample base level image using anti aliasing low pass filter at each level upto the highest level K. We get low resolution images as base level image.

The next step between two low resolution frames it computes displacement $d$ at each level of pyramid. First initial value of $\mathrm{d}=0$ is initialized for first two frame images $\mathrm{g} 1$ and $\mathrm{g} 2$ at all level K. It starts computing from upper level till down the lowest level of pyramid.

The advantage of Gaussian Pyramid Optical Flow is that it can reduce computing complex process and the algorithm can compute fast.

\subsection{PCA Registration Method}

The Principle Component Analysis (PCA) based Registration method is to high resolve image of a special (focused) part in an image. it can be obtains approximated shift, rotation and scale variance parameter. In that we firstly segment the focused part from the original image, and then binaries it with special part's pixel value as 1 and otherwise 0 ,where the special part's is called as BROI(Binary Region of Interest).Then, the PCA registration method measure the movement parameter and scale variance using the extracted BROI image and PCA. By segmenting human facial BROI from image, the color information can be achieving acceptable face region. In that first translating the RGB color space into YCC space and used the threshold value for using if a pixel belongs to human facial pixels. Then the morphological operators (for opening and closing processing) are used to the initial BROI face image for removing noise or filling the non-facial color.

The BROI part are needed to extract for all LR images have a same face, and measure a center $(\overline{\mathrm{X}}, \overline{\mathrm{Y}})$ of gravity of BROIs to estimate translation parameter between the reference image and the other images.

Then estimate rotation parameter and measure eigenvector of pixel coordinates covariance matrix $\mathrm{C}$ corresponding to gravity center in BROI using PCA and then, using PCA to calculate the Eigen value $\lambda$ and eigenvector e of symmetric covariance matrix $\mathrm{C}$ and the global orientation $\Theta$ of BROIs in all LR image. Then, lastly we the scale variance parameter obtained between the two BROI images by comparing the total pixel orientation between two BROIs images.PCA based registration method the BROIs from all low resolution images cannot be extracted precisely for minor image variance and a little noise.

\section{MULTIFRAME SUPER-RESOLUTION}

In this paper, Image registration using combination of PCA and GPOF method can give more accurate movement parameters. First we apply GPOF Registration, which builds Gaussian Pyramid for each frame of low resolution observed sequence. Then compute the optical flow $\mathrm{d}$ which is regarded as displacement from image $\mathrm{g} 2$ to $\mathrm{g} 1$ in each level of pyramid between two observed images. It starts computing from upper level till down the lowest level of pyramid.

Second, we can apply PCA Registration method. It is used to extract BROI from the image. Then morphological operators are applied on it. It is used for compression of image. It is used for precise registration.

Then image fusion is applied using simple averaging method to combine results of low resolution images. In this method the results of images are taken. Then they are added and the average is taken. So one output image is generated.

Then finally image interpolation using Bicubic interpolation is applied to get high resolved image. . In this method the interpolate point is replaced with the sixteen neighbouring pixel. The advantage of this method is simplicity and low computation. Then we get high resolved reconstructed image.

\section{EXPERIMENT RESULTS}

For the experiment results, we have to use the different quality measure parameters such as MSE, PSNR and SSIM to evaluate proposed work.

Mean square error ${ }^{[8]}$ is the squared error between the original image and reconstructed image. The MSE can be expressed as.

$$
M S E=\frac{1}{m n} \sum_{i=0}^{m-1} \sum_{i=0}^{n-1}[I(i, j)-K(i, j)]^{2}
$$

Where I and K represent the mxn matrices and also compare the images. They performed for the dimensions ' $i$ ' and ' $\mathrm{j}$ ' So, $I(i, j)$ represents the value of pixel $(i, j)$ of original image I and $K(i, j)$ represents the value of pixel $(i, j)$ of reconstructed image.

The Peak Signal-to-Noise Ratio (PSNR) ${ }^{[8]}$ is defined as a measuring of quality of reconstructed image and also comparing with original image. In that MSE is used for two mxn matrices represents with images I and $\mathrm{K}$ and compare the images. The PSNR can be expressed as.

$P S N R=20 \bullet \log _{10} \frac{M A x_{I}^{2}}{\sqrt{M S E}}$ 
Here, MAX $_{I}$ perform the maximum possible pixel value of the image. When the pixels are represented 8 bits per sample, this is 255.The PSNR expressed in decibels.

The structural similarity (SSIM) index ${ }^{[9]}$ was designed to better way the human visual system (HVS) processes structural information. SSIM measures structure of an image, contrast and compare variance and covariance between the two images. The SSIM can be expressed as:

$$
\operatorname{SSIM}(X, Y)=\frac{\left(2 \mu_{x} \mu_{y}+c_{1}\right)\left(2 \sigma_{x} \sigma_{y}+c_{2}\right)}{\left(\mu_{x}^{2}+\mu_{y}^{2}+c_{1}\right)\left(\sigma_{x}^{2}+\sigma_{y}^{2}+c_{2}\right)}
$$

Where, $\mathrm{x}$ and $\mathrm{y}$ are sub images of $\mathrm{X}$ and $\mathrm{Y} ; \mu \mathrm{x}, \mu \mathrm{y}$ are the average of $\mathrm{x}, \mathrm{y} ; \sigma \mathrm{x}, \sigma \mathrm{y}$ are standard deviations of $\mathrm{x}, \mathrm{y} . \mathrm{C} 1$ is set to $\mathrm{C} 1=(0.01 * 255)^{\wedge} 2$ and $\mathrm{C} 2=(0.01 * 255)^{\wedge} 2$.

We have considered for our experiment 4 input images like lenna,, butterfly, cameraman and parrot in Figure 1. The size of all 4 input images is $256 \mathrm{X} 256$.
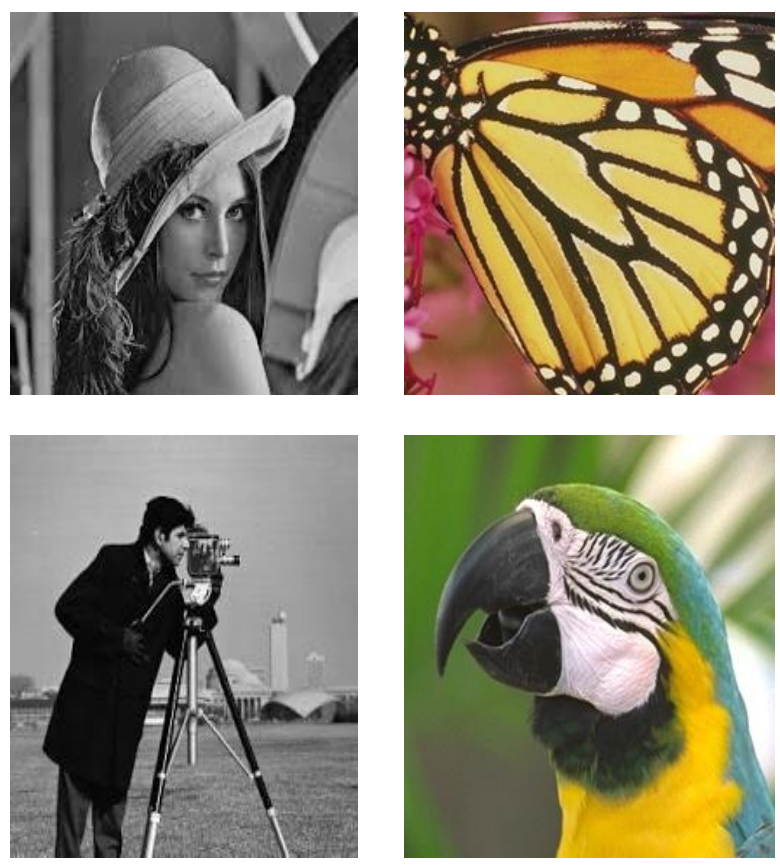

Figure 1: Input images with lenna, butterfly, cameraman and parrot

For the proposed algorithm, GPOF based registration method reduced the output images upto two levels. The GPOF based Regisration method using the 4 input images are shown in Figure 2 .
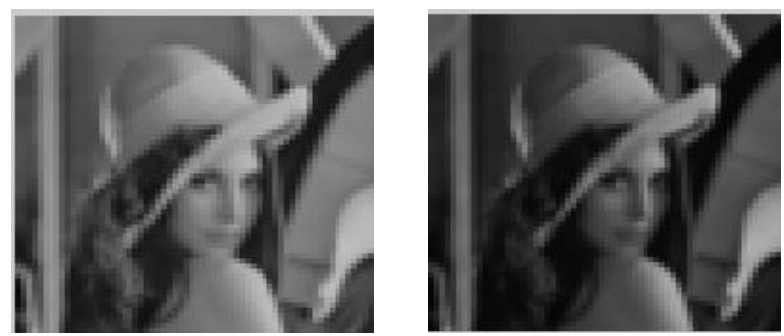
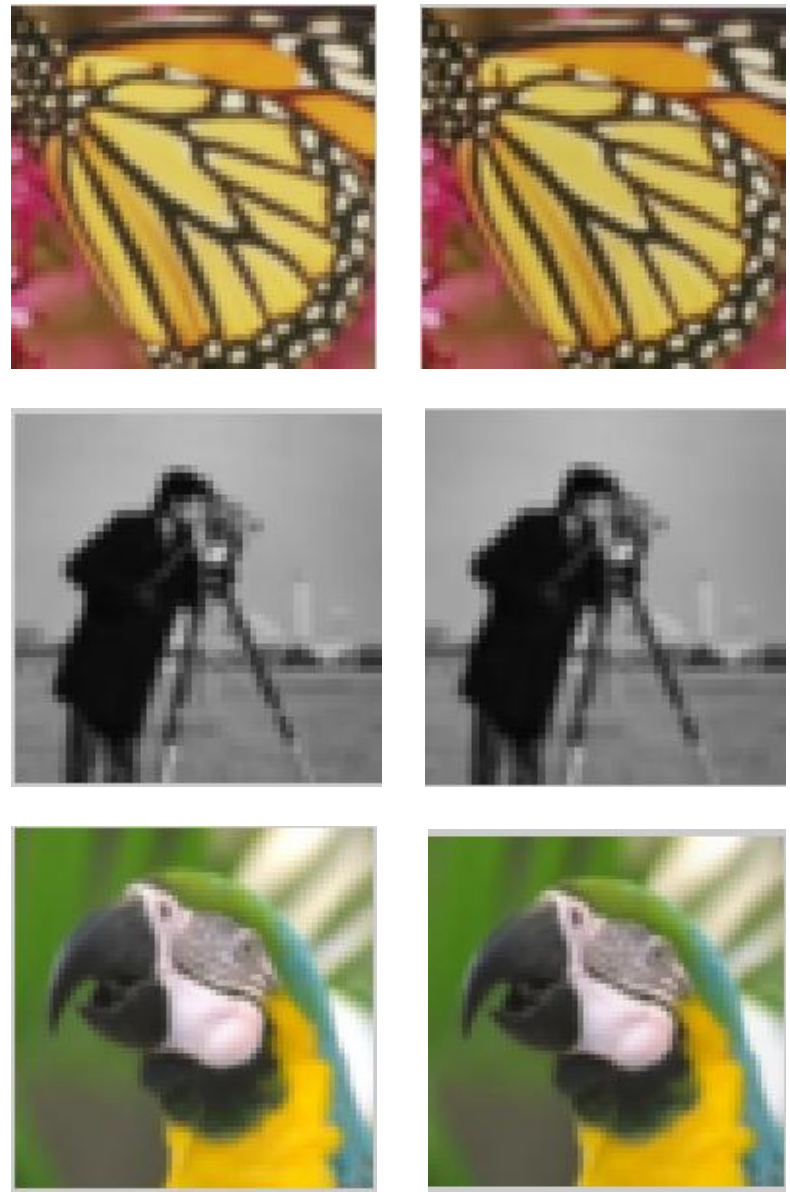

Figure 2: GPOF based registration method

For apply PCA based Registration method, the GPOF based Registration method's output are taken. The PCA based registration method and image fusion using simple averaging method's results are shown in Figure 3.
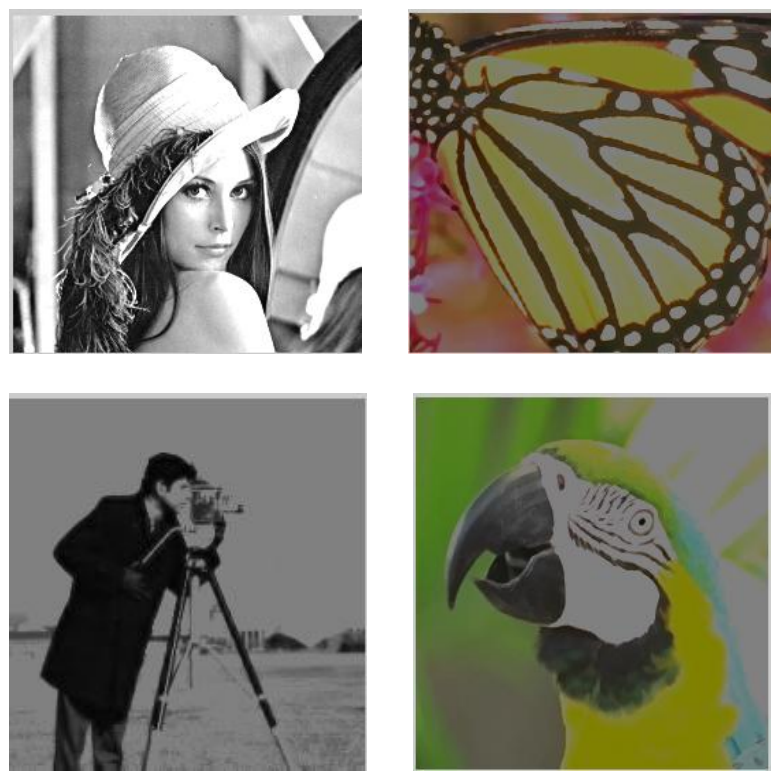

Figure 3: PCA based registration method and image fusion 
Lastly Image interpolation using Bicubic we get the High resolution or reconstructed image for all 4 input images. Figure 4 shows the High resolution or reconstructed images.
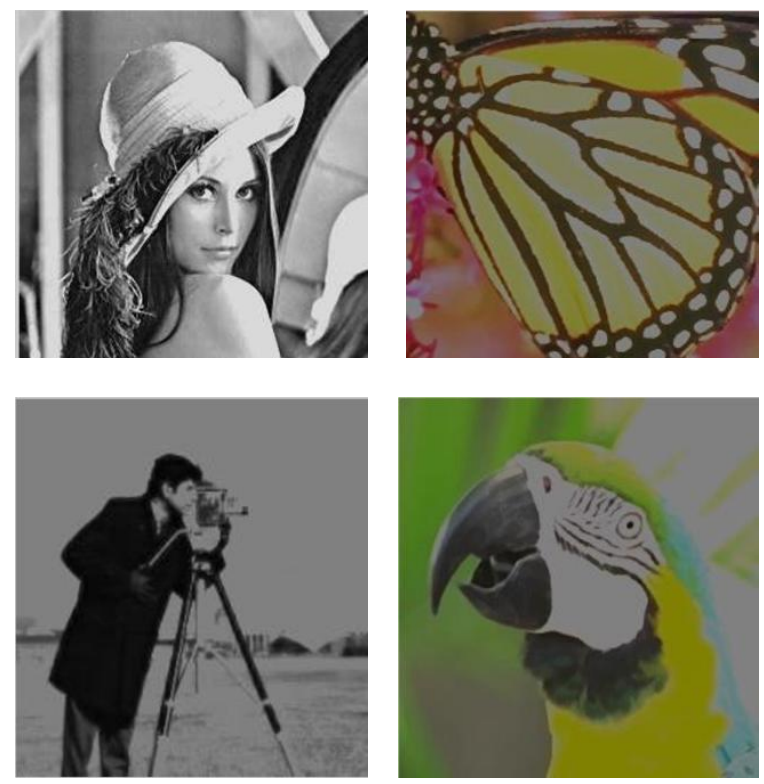

Figure 4: Reconstructed images and High resolution images

Image Quality measurement of MSE, PSNR and SSIM is defined between the original and reconstructed image. Experiment results of my proposed algorithm are shown in Table 1.

We compare two methods of Image Registration. Image quality measure using MSE,PSNR and SSIM and plot graph with input images and performance parameter. In Figure 5 shows the graph with input images Vs PSNR. The proposed results have been improved. In Figure 6 shows the graph of Input images Vs SSIM.

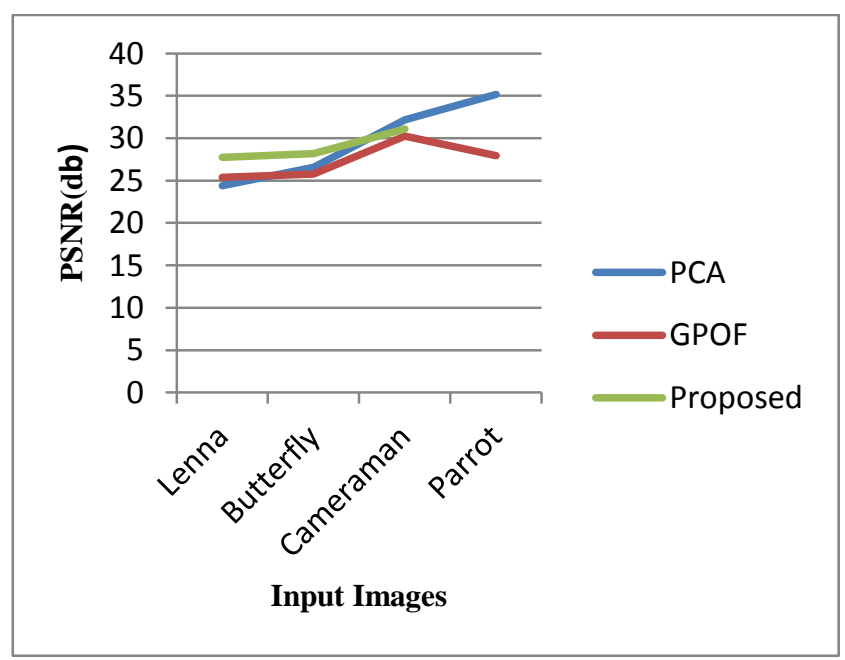

\begin{tabular}{|c|c|c|c|c|c|}
\hline \multirow[t]{2}{*}{ SR.NO } & \multirow[t]{2}{*}{ IMAGE } & \multirow[t]{2}{*}{ METHOD } & \multicolumn{3}{|c|}{$\begin{array}{l}\text { IMAGE QUALITY } \\
\text { MEASURE }\end{array}$} \\
\hline & & & MSE & PSNR & SSIM \\
\hline \multirow{3}{*}{1.} & \multirow{3}{*}{ Lenna } & PCA & 24.1024 & 24.3779 & 0.4487 \\
\hline & & GPOF & 49.8052 & 25.3792 & 0.3156 \\
\hline & & Proposed & 23.7627 & 27.7524 & 0.4360 \\
\hline \multirow{3}{*}{2.} & \multirow{3}{*}{ Butterfly } & PCA & 36.1268 & 26.5408 & 0.132 \\
\hline & & GPOF & 45.0927 & 25.7918 & 0.0891 \\
\hline & & Proposed & 28.7128 & 28.1647 & 0.1329 \\
\hline \multirow{3}{*}{3.} & \multirow{3}{*}{ Cameraman } & PCA & 35.1452 & 32.1578 & 0.3578 \\
\hline & & GPOF & 61.1471 & 30.2670 & 0.4449 \\
\hline & & Proposed & 36.9765 & 31.0815 & 0.4999 \\
\hline \multirow{3}{*}{4.} & \multirow{3}{*}{ Parrot } & PCA & 19.9153 & 35.1389 & 0.2099 \\
\hline & & GPOF & 57.1276 & 27.9168 & 0.1177 \\
\hline & & Proposed & 16.8423 & 35.1564 & 0.1567 \\
\hline
\end{tabular}

Table 1 Image Quality measurement of PSNR, MSE, SSIM

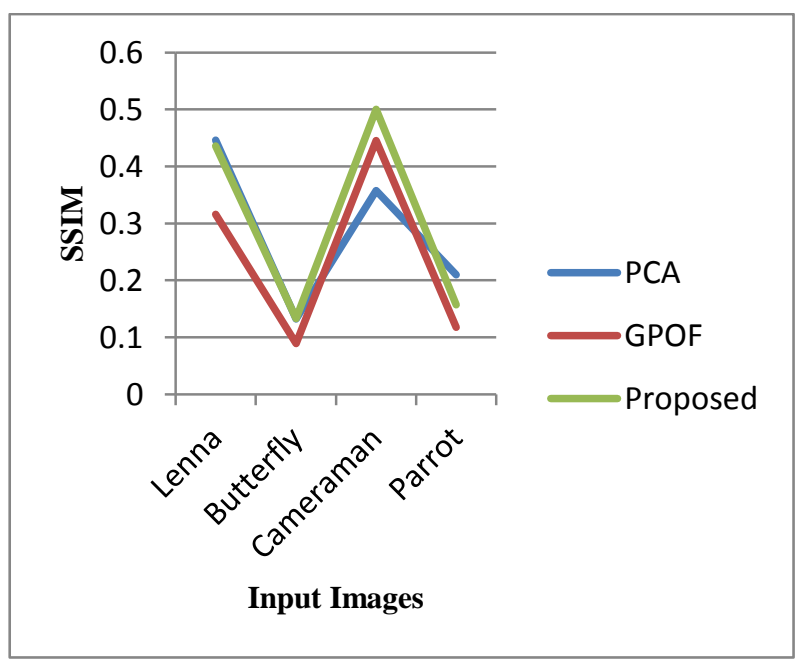

Figure 6: Input images vs. SSIM

Figure 5: Input images vs. PSNR 


\section{CONCLUSION}

This proposed technique is a combination of image registration methods PCA and GPOF for Multiframe superresolution. Our proposed GPOF registration is to measure the translation parameter and allows large pixel motion but keeping the image neighborhood relatively small. Then apply PCA registration method which can used for compression of image. Then Image Fusion is used to get one output image with multiple low resolution images. Finally image interpolation using Bicubic method is used to get High resolution or reconstructed image and we compare image registration methods with proposed method which achieves better results. We measure image quality using MSE,PSNR and SSIM indicate that proposed algorithm give better results.

\section{REFERENCES}

[1] S.C.Park, M.K.Park and M.G.Kang, "Super- resolution image reconstruction- A technical review"- IEEE Signal Processing Mag., vol. 20, pp. 21-36, May 2003.

[2] Xuelong Li,Yanting $\mathrm{Hu}$, Xinbo Gao, et.al, "A multiframe image super-resolution method", IEEE Transactions on Signal Processing 90, pp.405-414, 2010.

[3] Li Xin Na, Li Wen Ping, Wang Zheng Ming, Zhao Xia, "A Image Registration Method Combined with Gradient Similar Measure", IEEE Trans. Image Process., vol. 11, 2011.

[4] Lan Zhang; Hua Zhang; Simiao Zhang; YanbingXue, "Multi-frame image super-resolution reconstruction based on GPOF registration and L1-norm," Natural Computation (ICNC), 2010 Sixth International Conference on , vol.7, no., pp.3601,3604, 10-12 Aug. 2010

[5] Sasatani, S.; Xian-Hua Han; Yen-wei Chen, "Image registration using PCA and gradient method for superresolution imaging," Software Engineering and Data Mining (SEDM), 2010 2nd International Conference on , vol., no., pp.631,634, 23-25 June 2010.

[6] Kusum Rani ; Reecha Sharma, "Srudy of Different Image fusion Algorithm", International journal of Emerging Technology and advanced engineering,2013.

[7] Chidananda Murthy, M.V.; Yallapurmath, V.; Kurian, M. Z.; Guruprasad, H. S., "Design and implementation of interpolation algorithms for image super resolution," Communication Systems, Networks \& Digital Signal Processing (CSNDSP), 2012 8th International Symposium on, vol., no., pp.1,6, 18-20 July 2012.

[8] Z. Wang, A. C. Bovik, H. R. Sheikh and E. P. Simoncelli, "Image quality assessment: From error visibility to structural similarity", IEEE Trans. Image Process., vol. 13, no. 4, pp. 600-612, 2004.

[9] Yusra A.Y. Al-Najjar, Dr. Der Chen Soong, "Comparison of Image Quality Assessment: PSNR, HVS, SSIM, UIQI”, International Journal of Scientific \& Engineering Research, Volume 3, Issue 8, August2012.

[10] Simon Baker, Takeo Kanade, ”Super-resolution optical flow", Technical Report CMU.

[11] Nan Zhao; Cuihua Li; Hua Shi; Chen Lin, "Multi-Frame Image Super-Resolution Based on Regularization Scheme," Control, Automation and Systems Engineering (CASE), 2011 International Conference on , vol., no., pp.1,4, 30-31 July 2011.

[12] S. Farsiu, M. D. Robinson, M. Elad, and P. Milanfar, "Fast and robust Multi-frame super resolution", IEEE Trans. Image Process., vol. 13, no.10, pp. 1327-1344, 2004.

[13] R. Tsai, T. Huang,"Multi-frame image restoration and registration, in: Advances in Computer Vision and Image Processing, vol. 1, no. 2, JAI Press Inc., Greenwich, CT, 1984, pp. 317-339.

[14] Vishal R. Jaiswal, Suhas H. Patil, Girish P. Potdar, ShrishailT.Patil," Multi-frame Image Super-ResolutionA Comparison",(IJCSE) International Journal On Computer Science and Engineering, Vol. 02, No. 09, 2010.

[15] Turgay Celik, Kai-Kuang Ma, "Fast Object-based Image Registration Using Principal Component Analysis for Super-resolution Imaging", 5th International Conference on Visual Information Engineering, pp.705-710, 2008.

[16] Nelson, K.; Bhatti, A.; Nahavandi, S., "Performance Evaluation of Multi-Frame Super-Resolution Algorithms," Digital Image Computing Techniques and Applications (DICTA), 2012 International Conference on pp.1,8, 3-5 Dec. 2012. 